\title{
Novel Methods for Assessing Oral Direct Factor $X a$ and Thrombin Inhibitors: Use of Point-of-Care Testing and Urine Samples
}

\author{
Job Harenberg, MD, $\mathrm{PhD}^{1}$ Shanshan Du, MSc ${ }^{1}$ Sandra Krämer, BSc ${ }^{1} \quad$ Christina Giese, FRBTech ${ }^{1}$ \\ Astrid Schulze, $\mathrm{MD}^{2}$ Christel Weiss, $\mathrm{PhD}^{3}$ Roland Krämer, $\mathrm{PhD}^{4}$
}

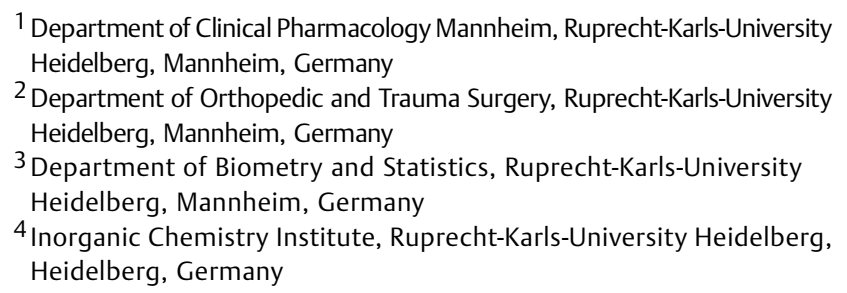

Address for correspondence and reprint requests Job Harenberg, MD, PhD, Department of Clinical Pharmacology Manheim, Ruprecht-Karls-University Heidelberg, Maybachstr. 14, 68169 Mannheim, Germany (e-mail: job.harenberg@medma.uni-heidelberg.de).

Semin Thromb Hemost 2013;39:66-71.

\author{
Abstract \\ Keywords \\ - new oral \\ anticoagulants \\ - dabigatran \\ - rivaroxaban \\ - point-of-care method \\ - urine
}

Rivaroxaban and dabigatran are new oral anticoagulants (NOACs) that inhibit directly factor Xa and thrombin, respectively. These NOACs effectively prevent thromboembolic complications using fixed doses without the need for dose adjustment according to laboratory results. About $60 \%$ of rivaroxaban is cleared from circulation by glomerular filtration, $30 \%$ of which is excreted as active drug. About $80 \%$ of dabigatran is excreted into urine as active compound. Accordingly, both NOACs can be determined in urine by means of chromatographic methods. Only a few laboratories are able to perform such methods, and results are not available within short time frames. New methods have to be developed to obtain results within minutes and possibly as point-of-care (POC) techniques. This testing may be useful for special patient populations such as those with acute deterioration of renal function due to any disease, before surgical interventions, during unexpected bleeding or thrombotic episodes while on therapy with NOACs, the oldest and youngest populations, pregnancy, suspicion of overdose and intoxication, and to determine adherence to therapy. Here we describe results of a POC qualitative assay using urine samples from patients on treatment with dabigatran and rivaroxaban.
Thromboembolism is one of the major complications after primary elective total hip and knee replacement surgery (resulting in considerable preventable morbidity and mortality) ${ }^{1}$ and in patients with nonvalvular atrial fibrillation (AF; leading to cerebral ischemic stroke and systemic embolism). ${ }^{2}$ Limitations of the conventional regimes for prophylaxis of venous thromboembolism with low-molecular-weight heparin (LMWH) include bleeding hematoma, local allergy, heparin-induced thrombocytopenia type I and type II, transient increase of liver enzymes, and the requirement for parenteral administration. $^{3}$ Anticoagulation in patients with AF using vitamin $\mathrm{K}$ antagonists (VKA) reduces the incidence of ischemic stroke and systemic embolism and mortality, but severe bleeding complications, including intracranial hemorrhage, may occur. ${ }^{4}$ Also, VKA usage requires frequent dose adjustments to maximize the time in the therapeutic range of international normalized ratio values between 2 and 3.,6 Many interactions with food and drugs and the slow onset and offset of action of VKAs necessitate simultaneous administration of unfractionated heparins or LMWHs during induction of anticoagulation as well as during discontinuation of anticoagulation. The new oral anticoagulants (NOACs) have
Issue Theme Hot Topics IV; Guest Editor, Emmanuel J. Favaloro, PhD, FFSc (RCPA). published online December 27, 2012
DOI http://dx.doi.org/ 10.1055/s-0032-1331155. ISSN 0094-6176.
Copyright (c) 2013 by Thieme Medical Publishers, Inc., 333 Seventh Avenue, New York, NY 10001, USA. Tel: +1(212) 584-4662.
License terms

()ㅇㅇ $\Theta \circledast$ 
thereby been developed to overcome most of these drawbacks of heparins and VKAs and to help improve patient care. ${ }^{7}$

The oral direct factor Ila inhibitor dabigatran and the oral direct factor Xa inhibitors rivaroxaban and apixaban have been approved in patients for prevention of venous thromboembolism in total hip and total knee replacement surgery. Dabigatran and rivaroxaban have received approval for prevention of embolism in patients with $\mathrm{AF}$ and rivaroxaban for treatment of venous thromboembolism, pulmonary embolism, and prevention of recurrent events. Differences of doses exist for some of the NOACs between the approval by the U.S. Food and Drug Administration and the European Medicinal Agency. Approvals for additional indications and for other NOACs are pending.

One of the advantages of the NOACs such as rivaroxaban and dabigatran is use of a daily fixed dosage without laboratory-guided drug monitoring. ${ }^{8}$ Despite this, the amount of anticoagulant present in a given individual may need to be determined in several clinical situations. The need of determination of anticoagulant effects is increasingly evident for patients with deterioration of renal function, in the elderly or oldest population, for surgery or in acute clinical situations, during recurrent events or during bleeding complications, or to check compliance. ${ }^{9,10}$ The concentration of NOACs can be accurately determined by high-pressure liquid chromatography methods. These techniques are, however, unsuitable for clinical routine use. Thrombin and factor Xa-dependent coagulation or chromogenic substrate assays have therefore been developed to respectively determine the concentration or activity of dabigatran and rivaroxaban. ${ }^{11,12}$ However, point-of-care (POC) methods to measure NOACs have not been reported so far.

POC methods are useful tools to improve patient care and are available for assessment of several cardiovascular biomarkers. POC methods for cardiac troponin T, N-terminal probrain natriuretic peptide, ${ }^{13}$ and D-dimer are the most used, in particular for cardiovascular risk stratification. ${ }^{14}$ The clinical value of D-dimer, an indicator of fibrin degradation and coagulation activation, lies in its high negative predictive value for excluding deep vein thrombosis and pulmonary embolism..$^{15}$ Other POC methods are available for assessment of genetic defects of blood coagulation factors resulting in an increased thromboembolic risk. ${ }^{16}$ In blood coagulation, POC testing is normally performed for control of treatment with $\mathrm{VKA},{ }^{17}$ for control of antiplatelet therapy, ${ }^{18}$ and for assessment of coagulation using thrombelastography. ${ }^{19}$

NOACs are excreted into the urine, up to $66 \%$ for rivaroxaban, $30 \%$ of which as active form,,$^{20}$ and up to $80 \%$ for dabigatran, all of which as the active form of the parent compound. ${ }^{21}$ The NOACs can be determined in urine by highpressure liquid chromatography methods as in plasma samples, but these methods are complex and not generally available. Easier to perform methods have also recently been described to determine these anticoagulants in urine from patients on treatment. However, assessment may also be difficult to interpret because the concentration depends on the time between intake of the drug and urine sampling. Advantages of urine sampling include the non-invasive tech- niques and the possibility to develop POC test systems. These assays can be performed by trained personal or by the patients themselves, and can be easily repeated. Here we describe the results of a validation of POC qualitative tests for dabigatran and rivaroxaban using urine samples from patients on treatment and compared with the results obtained using plasma samples.

\section{Methods and Patients}

\section{Analysis of Dabigatran}

\section{Urine Samples}

In urine, dabigatran was determined by a POC method incubating the lyophilized reagents on ministrips followed by incubation with patient's urine ${ }^{22}$ (International Patent Application No. PCT/EP2012/002540). During the incubation of the strips with urine, the blue color of the solution changes to a green color in the absence of dabigatran within 15 minutes. In the presence of dabigatran in urine the color remains blue after 15 minutes of incubation. The reaction is stopped with acetic acid, and the color is identified by eye and is documented photographically.

\section{Plasma Samples}

The chromogenic substrate S2238 (Instrumentation Laboratory $\mathrm{GmbH}$, Kirchheim, Germany) was used to determine dabigatran in plasma. ${ }^{23}$ Standard curves were computed using serial concentrations of dabigatran ranging from 25 to $500 \mathrm{ng} / \mathrm{mL}$ added to normal pool plasma. The assay was performed as described previously. ${ }^{24}$

\section{Origin and Quality of Dabigatran}

Dabigatran was purified from commercially available Pradaxa (Dabigatran etexilate, Boehringer Ingelheim Pharma, Ingelheim, Germany), and its purity was characterized by analytical methods. ${ }^{24}$ The thrombin inhibition capacity was determined by a S2238 thrombin-specific chromogenic substrate assay in comparison with the hemoclot assay containing dabigatran from Boehringer Ingelheim (Hyphen BioMed, Neuville-sur-Oise, France). The correlation of the concentration of dabigatran by these two assays was $r=0.6733$ $(p<0.01){ }^{24}$

\section{Patients on Treatment with Dabigatran}

Urine and plasma samples were taken from patients with nonvalvular AF on treatment with 110 or $150 \mathrm{mg}$ dabigatran twice daily $(n=110)$ and healthy persons $(n=112)$ after having given written informed consent.

\section{Preparation and Analysis of Samples}

\section{Urine Samples}

Urine samples were taken from control subjects, aliquoted, centrifuged at $1,800 \times \mathrm{g}$ and $4^{\circ} \mathrm{C}$, shock frozen, and stored at $-72^{\circ} \mathrm{C}$ until analysis. Urine samples from patients were taken 2 hours after intake of dabigatran and also processed as described above. Samples were later thawed and incubated with strips containing the reagents for 15 minutes, and the 
color was read at $405 \mathrm{~mm}$ and by eye and documented photographically.

\section{Plasma Samples}

Blood samples were taken from non-anticoagulated control subjects, and from patients 2 hours after intake of the anticoagulant into plastic tubes containing $3.8 \%$ sodium citrate (v/v, 1/10, citrate/plasma), centrifuged within 30 minutes at $1,800 \times \mathrm{g}$ and $4^{\circ} \mathrm{C}$, and the plasma supernatant was shock frozen, and kept at $-72^{\circ} \mathrm{C}$ until analysis.

\section{Sensitivity and Specificity}

\section{Urine Samples}

The sensitivity and specificity of the qualitative reading of the color of urine samples were calculated from control samples and samples from patients on treatment with dabigatran, respectively. The green color represented a negative result, meaning the samples did not contain dabigatran. The blue color of the solution represented the presences of dabigatran.

\section{Kappa Index and Youden Index}

Color development was read by three skilled persons independently to determine the kappa coefficient of the interreader agreement. ${ }^{25,26}$ To determine the diagnostic test accuracy the Youden index (Youden $=$ sensitivity + specificity -1 ) was used, which is preferable to the diagnostic odds ratio. ${ }^{27}$ The Youden index was determined for the urine samples using the results by judgment of the color by the three independent readers.

\section{Analysis of Rivaroxaban}

\section{Urine Samples}

Control urine samples were taken before administration of $10 \mathrm{mg}$ rivaroxaban once daily from patients admitted for primary elective knee or hip replacement surgery. The second urine sample was collected from every patient 12 hours after 4 to 6 days of daily intake of rivaroxaban in the evening.

\section{Preparation and Analysis of Samples}

Urine and plasma samples were obtained and processed as described previously for dabigatran.

\section{Urine Samples}

In urine, rivaroxaban was determined by a POC method incubating the lyophilized reagents on strips followed by incubation with patient's urine over 15 minutes ${ }^{28}$ (international patent PCT/WO2012/069139A1). The yellow color of the urine itself is diluted with the reagents to a transparent solution. If the urine does not contain rivaroxaban, a yellow color develops during incubation with the reagents immobilized on the strips. If the urine contains rivaroxaban, no color develops and the solution remains clear.

\section{Plasma Samples}

The S2222 chromogenic substrate assay ( $N$-benzoyl-L-isoleucyl-L-glutamylglycyl-L-arginine-p-nitroaniline hydrochlo- ride and its methyl ester, Instrumentation Laboratory $\mathrm{GmbH}$, Kirchheim, Germany) was used to determine rivaroxaban in plasma as described. $^{29}$

\section{Source of Rivaroxaban as Standard Solution for Assays} Rivaroxaban was purified from commercially available Xarelto (Rivaroxaban, Bayer HealthCare, Wuppertal, Germany). The purity and anti-factor Xa activity were described earlier. ${ }^{30}$ Standard solutions were prepared for determination of rivaroxaban in urine and plasma. Serial concentrations of rivaroxaban ranging from 12.5 to $500 \mathrm{ng} / \mathrm{mL}$ were added to pool plasma obtained from 20 healthy donors. Blood samples were collected as described above to obtain and store plasma samples.

\section{Patients on Treatment with Rivaroxaban}

Urine and plasma samples were taken from patients ( $n=144)$ after having given written informed consent before and once at days 4 to 6 after total knee replacement and total hip replacement surgery on antithrombotic therapy with $10 \mathrm{mg}$ daily rivaroxaban.

\section{Sensitivity and Specificity of the Assays}

The sensitivity of the urine and plasma assays and the intereader agreement and the diagnostic test accuracy were determined as described above.

\section{Results}

\section{Dabigatran}

Urine Samples

Using the POC method for dabigatran in urine, all 112 control persons had negative values by eye judgment ( - Table 1 ).

During therapy with dabigatran, all 110 patients had positive color development as judged by eye. Accordingly, the sensitivity, specificity, and accuracy were $100 \%$ (-Table 1). The Youden index was 1 . The interrater agreement was 1.0 of the three independent persons.

\section{Plasma Samples}

Control patients displayed plasma concentrations of $40 \pm 30$ $\mathrm{ng} / \mathrm{mL}$. In patients on therapy the concentration of dabigatran was $120 \pm 80 \mathrm{ng} / \mathrm{mL}(n=110)$.

\section{Rivaroxaban}

\section{Urine Samples}

Using the POC method for rivaroxaban in urine before start of therapy (available samples 117 of 144 patients), all 117 patients had negative values as judged by eye.

During therapy with rivaroxaban, 4 of 125 of 144 available samples showed a yellow color development by eye measurement. One hundred twenty-one of 125 patients showed no color development as judged by eye. The sensitivity was $96.8 \%$, the specificity $100 \%$, and the accuracy $98.3 \%$ (-Table 2). The Youden index of the interrater agreement was $96.8 \%$. 
Table 1 Dabigatran in urine

\begin{tabular}{|l|l|l|}
\hline & Control & During therapy \\
\hline $\begin{array}{l}\text { Positive: } \\
\text { blue color }(n)\end{array}$ & 0 & 110 \\
\hline$\%$ & $\begin{array}{l}\text { False positive } \\
\%\end{array}$ & $\begin{array}{l}\text { Correct positive } \\
100\end{array}$ \\
\hline $\begin{array}{l}\text { Negative: } \\
\text { green color }(n)\end{array}$ & 112 & 0 \\
\hline$\%$ & $\begin{array}{l}\text { Correct negative } \\
100\end{array}$ & $\begin{array}{l}\text { False negative } \\
0\end{array}$ \\
\hline Sum & 112 & 110 \\
\hline
\end{tabular}

Note: Results of the determination of dabigatran in urine samples of patients not taking any anticoagulant (control) and 4-6 months on treatment 2 hours after intake of dabigatran 110 or $150 \mathrm{mg}$ twice daily (samples during therapy available only from 110 patients). Data are given as negative and positive according to the color visible to the naked eye.

\section{Plasma Samples}

Patients displayed plasma concentrations of $11.1 \pm 7.1 \mathrm{ng} / \mathrm{mL}$ before administration of rivaroxaban. During therapy the concentration of rivaroxaban was $76.7 \pm 36.6 \mathrm{ng} / \mathrm{mL}$.

\section{Discussion}

The results of the investigations demonstrate that the POC method from urine samples of patients on treatment with dabigatran and rivaroxaban displays a specificity of $100 \%$. The sensitivity and accuracy are $100 \%$ for dabigatran and about $97 \%$ for rivaroxaban. The test was developed as a qualitative determination for the presence or absence of the anticoagulants. Therefore, negative and positive results can be judged by eye with different colors. Negative and positive detections of dabigatran were related to green and blue color of the solution of the POC test and yellow and no color for the absence or presence of rivaroxaban, respectively. The interrater agreement is relevant because the readers have to decide which color they see by eye. The interrater agreement was 1.0 for dabigatran and 0.97 for rivaroxaban samples.

Table 2 Rivaroxaban in urine samples

\begin{tabular}{|l|l|l|}
\hline & Control & During therapy \\
\hline $\begin{array}{l}\text { Positive: } \\
\text { no color }(n)\end{array}$ & 0 & 121 \\
\hline$\%$ & $\begin{array}{l}\text { False positive } \\
0\end{array}$ & $\begin{array}{l}\text { Correct positive } \\
96.8\end{array}$ \\
\hline $\begin{array}{l}\text { Negative: } \\
\text { yellow color }(n)\end{array}$ & 117 & 4 \\
\hline & $\begin{array}{l}\text { Correct negative } \\
\%\end{array}$ & $\begin{array}{l}\text { False negative } \\
3.2\end{array}$ \\
\hline Sum $(N)$ & 100 & 125 \\
\hline
\end{tabular}

Note: Results of the determination of rivaroxaban in urine samples of patients not taking any anticoagulant (control) and 4-6 days on treatment 12 hours after intake of rivaroxaban $10 \mathrm{mg}$ once daily (samples available only from $117 / 144$ patients before and $125 / 144$ patients during therapy). Data are given as negative and positive according to the color visible to eye.
NOACs are given in fixed doses once or twice daily without need for laboratory-guided dose adjustment. However, certain clinical situations may require the determination of their anticoagulant effect. These situations may occur more frequently in acute than in chronic situations. POC methods are available and are successfully used for determination of the international normalized ratio during treatment with VKA. At present, the thromboplastin reagents used for the currently available coagulation POC monitors are not sensitive toward rivaroxaban or dabigatran. ${ }^{31}$ In chronic therapy for many diseases, adherence to drug treatment was shown to increase by using POC methods. ${ }^{32}$ POC methods from urine samples have been reported for several drugs ${ }^{33}$ and have similarly been shown to improve adherence to therapy. ${ }^{34}$ Urine samples are easy to collect and are commonly used to control adherence to drug therapy. The patient and any health care personal may collect urine samples. If samples are analyzed by a POC system, results are available within minutes.

Chronic disease is a common presentation in general practice. Improving long-term medication adherence is crucial to improving chronic disease outcomes. Evidence from large-scale, randomized, controlled trials is that medication adherence is suboptimal, ${ }^{35,36}$ which is influenced by many variables, including number and cost of medications, duration of disease, age, and patient attitudes, beliefs, and perceptions relating to illness. ${ }^{37,38}$ Factors that positively influence patients' outcomes include active patient involvement in decision-making processes affecting their care, good communication, and providing patients with education and quality information about their disease. ${ }^{39,40}$ POC testing produces an immediate test result, thus enabling immediate decisions and discussion about patient treatment, and has the potential to engage patients in their own care. A systematic review of POC testing by Delaney and colleagues suggested that these methods may be valuable in managing patients with chronic conditions. ${ }^{41}$ This indicates the potential of POC methods for the NOACs dabigatran, rivaroxaban, apixaban, and others in development.

The strengths of the methods are that they are not invasive, they are repetitively easy to perform, the results are available within 15 minutes, they can be performed by patients themselves, and they do not require standard solutions. The methods are highly sensitive, specific, accurate, and posses a very high interrater agreement.

Limitations of the POC methods include the lack of precise data on the pharmacokinetics in relation to the urinary excretion of dabigatran and rivaroxaban over time. Another limitation refers to severe renal impairment. The NOACs may not be excreted into urine in those patients. Urine assessment of the NOACs does not necessarily reflect the acute concentration of the drug but rather its concentration in the previous hours (i.e., from the last urine produced). This is particularly essential for emergency physicians, who should be aware of how to interpret results. However, acute urine samples can be repetitively obtained in emergency situations to cover this problem.

In conclusion, the POC methods using urine samples from patients on treatment with dabigatran and rivaroxaban are 
characterized by a very high sensitivity, specificity, and accuracy. The POC method is noninvasive, easy to perform, and can be done repetitively. The validity of these methods in pathologic urine samples and the real world use by patients remain to be investigated.

\section{Acknowledgment}

The research was supported by a grant of the Dietmar Hopp Foundation. We acknowledge the technical assistance of Mrs. Christina Giese, Antje Hagedorn, Inge Träger, and Ulrich Warttinger.

\section{References}

1 Falck-Ytter Y, Francis CW, Johanson NA, et al. Prevention of VTE in orthopedic surgery patients: antithrombotic therapy and Prevention of thrombosis, 9th ed: American College of Chest Physicians Evidence-Based Clinical Practice Guidelines. Chest 2012;141:(2) (Suppl):e278S-e325S

2 Roskell NS, Lip GY, Noack H, Clemens A, Plumb JM. Treatments for stroke prevention in atrial fibrillation: a network meta-analysis and indirect comparisons versus dabigatran etexilate. Thromb Haemost 2010;104(6):1106-1115

3 Linkins LA, Dans AL, Moores LK, et al. Treatment and Prevention of Heparin-Induced Thrombocytopenia: Antithrombotic Therapy and Prevention of Thrombosis, 9th ed: American College of Chest Physicians Evidence-Based Clinical Practice Guidelines. Chest 2012;141:e495S-530S

4 Lip GY, Andreotti F, Fauchier L, et al; European Heart Rhythm Association. Bleeding risk assessment and management in atrial fibrillation patients. Executive Summary of a Position Document from the European Heart Rhythm Association [EHRA], endorsed by the European Society of Cardiology [ESC] Working Group on Thrombosis. Thromb Haemost 2011;106(6):997-1011

5 Schmitt L, Speckman J, Ansell J. Quality assessment of anticoagulation dose management: comparative evaluation of measures of time-intherapeutic range. J Thromb Thrombolysis 2003;15(3):213-216

6 Lader E, Martin N, Cohen G, et al. Warfarin therapeutic monitoring: is $70 \%$ time in the therapeutic range the best we can do? J Clin Pharm Ther 2012;37(4):375-377

7 Harenberg J, Marx S, Krejczy M, Wehling M. New anticoagulants promising and failed developments. Br J Pharmacol 2012;165 (2):363-372

8 Ten Cate H. Monitoring new oral anticoagulants, managing thrombosis, or both? Thromb Haemost 2012;107(5):803-805

9 Mismetti P, Laporte S. New oral antithrombotics: a need for laboratory monitoring. For. J Thromb Haemost 2010;8(4): 621-626

10 Harenberg J, Marx S, Erdle S, Krämer R. Determination of the anticoagulant effects of new oral anticoagulants: an unmet need. Expert Rev Hematol 2012;5(1):107-113

11 Favaloro EJ, Lippi G, Koutts J. Laboratory testing of anticoagulants: the present and the future. Pathology 2011;43(7):682-692

12 Favaloro EJ, Lippi G. Laboratory testing and/or monitoring of the new oral anticoagulants/antithrombotics: for and against? Clin Chem Lab Med 2011;49(5):755-757

13 Anderson JL, Adams CD, Antman EM, et al; American College of Cardiology; American Heart Association Task Force on Practice Guidelines (Writing Committee to Revise the 2002 Guidelines for the Management of Patients With Unstable Angina/Non-ST-Elevation Myocardial Infarction); American College of Emergency Physicians; Society for Cardiovascular Angiography and Interventions; Society of Thoracic Surgeons; American Association of Cardiovascular and Pulmonary Rehabilitation; Society for Aca- demic Emergency Medicine. ACC/AHA 2007 guidelines for the management of patients with unstable angina/non ST-elevation myocardial infarction: a report of the American College of Cardiology/American Heart Association Task Force on Practice Guidelines (Writing Committee to Revise the 2002 Guidelines for the Management of Patients With Unstable Angina/Non ST-Elevation Myocardial Infarction): developed in collaboration with the American College of Emergency Physicians, the Society for Cardiovascular Angiography and Interventions, and the Society of Thoracic Surgeons: endorsed by the American Association of Cardiovascular and Pulmonary Rehabilitation and the Society for Academic Emergency Medicine. Circulation 2007;116(7):e148-e304

14 Tomonaga Y, Gutzwiller F, Lüscher TF, et al. Diagnostic accuracy of point-of-care testing for acute coronary syndromes, heart failure and thromboembolic events in primary care: a cluster-randomised controlled trial. BMC Fam Pract 2011;12:12

15 Sidelmann JJ, Gram J, Larsen A, Overgaard K, Jespersen J. Analytical and clinical validation of a new point-of-care testing system for determination of D-dimer in human blood. Thromb Res 2010;126 (6):524-530

16 Van Cott EM. Point-of-care testing in coagulation. Clin Lab Med 2009;29(3):543-553

17 Jespersen J, Poller L, van den Besselaar AM, et al. External quality assessment (EQA) for CoaguChek monitors. Thromb Haemost 2010;103(5):936-941

18 Chen F, Maridakis V, O'neill EA, et al. A randomized clinical trial comparing point-of-care platelet function assays and bleeding time in healthy subjects treated with aspirin or clopidogrel. Platelets 2012;23(4):249-258

19 Görlinger K, Dirkmann D, Hanke AA, et al. First-line therapy with coagulation factor concentrates combined with point-of-care coagulation testing is associated with decreased allogeneic blood transfusion in cardiovascular surgery: a retrospective, singlecenter cohort study. Anesthesiology 2011;115(6):1179-1191

20 Kubitza D, Becka M, Mueck W, et al. Effects of renal impairment on the pharmacokinetics, pharmacodynamics and safety of rivaroxaban, an oral, direct Factor Xa inhibitor. Br J Clin Pharmacol 2010; 70(5):703-712

21 Poulsen BK, Grove EL, Husted SE. New oral anticoagulants: a review of the literature with particular emphasis on patients with impaired renal function. Drugs 2012;72(13):1739-1753

22 Harenberg J, Kraemer R. Direct thrombin inhibitors. International Patent Application No. PCT/EP2012/002540

23 Gray E, Harenberg J; ISTH Control of Anticoagulation SSC Working Group on Thrombin Inhibitors. Collaborative study on monitoring methods to determine direct thrombin inhibitors lepirudin and argatroban. J Thromb Haemost 2005;3(9):2096-2097

24 Harenberg J, Giese C, Marx S, Krämer R. Determination of dabigatran in human plasma samples. Semin Thromb Hemost 2012;38 (1):16-22

25 Feinstein AR, Cicchetti DV. High agreement but low kappa: I. The problems of two paradoxes. J Clin Epidemiol 1990;43(6):543-549

26 Hasnain M, Onishi H, Elstein AS. Inter-rater agreement in judging errors in diagnostic reasoning. Med Educ 2004;38(6):609-616

27 Böhning D, Holling H, Patilea V. A limitation of the diagnostic-odds ratio in determining an optimal cut-off value for a continuous diagnostic test. Stat Methods Med Res 2011;20(5):541-550

28 Harenberg J, Kraemer R. Direct factor Xa inhibitors. International Patent No. PCT/EP2011/WO2012/06913A1

29 Harenberg J, Erdle S, Marx S, Krämer R. Determination of rivaroxaban in human plasma samples. Semin Thromb Hemost 2012;38 (2):178-184

30 Harenberg J, Marx S, Weiss C, Krämer R, Samama M, Schulman S; on behalf of the working party: methods to determine rivaroxaban of the Subcommittee on Control of Anticoagulation of the ISTH. Report of the Subcommittee of Control of Anticoagulation on the determination of the anticoagulant effects of rivaroxaban. J Thromb Haemost 2012;10(7):1433-1436 
31 Tripodi A. Problems and solutions for testing hemostasis assays while patients are on anticoagulants. Semin Thromb Hemost 2012;38(6):586-592

32 Mahler C, Hermann K, Horne R, et al. Assessing reported adherence to pharmacological treatment recommendations. Translation and evaluation of the Medication Adherence Report Scale (MARS) in Germany. J Eval Clin Pract 2010;16(3):574-579

33 Attema-de Jonge ME, Peeters SY, Franssen EJ. Performance of three point-of-care urinalysis test devices for drugs of abuse and therapeutic drugs applied in the emergency department. J Emerg Med 2012;42(6):682-691

34 Cervinski MA, Lockwood CM, Ferguson AM, et al. Qualitative point-of-care and over-the-counter urine hCG devices differentially detect the hCG variants of early pregnancy. Clin Chim Acta 2009;406(1-2):81-85

35 Cramer JA. A systematic review of adherence with medications for diabetes. Diabetes Care 2004;27(5):1218-1224
36 Huser MA, Evans TS, Berger V. Medication adherence trends with statins. Adv Ther 2005;22(2):163-171

37 Burkhart PV, Sabaté E. Adherence to long-term therapies: evidence for action. J Nurs Scholarsh 2003;35(3):207

38 Horne R, Weinmann J, Barber N. Concordance, Adherence and Compliance in Medicine Taking. London, England: National Coordinating Centre for NHS Service Delivery and Organisation Research and Development (NCCDSO); 2005

39 Tabrizi JS, Wilson AJ, Coyne ET, O'Rourke PK. Clients' perspective on service quality for type 2 diabetes in Australia. Aust N Z J Public Health 2007;31(6):511-515

40 Kaplan SH, Greenfield S, Ware JE Jr. Assessing the effects of physician-patient interactions on the outcomes of chronic disease. Med Care 1989;27(3, Suppl):S110-S127

41 Delaney BC, Hyde CJ, McManus RJ, et al. Systematic review of near patient test evaluations in primary care. BMJ 1999;319 (7213):824-827 